\title{
COMPOSIÇÃO ACARINA EM DIFERENTES CULTIVARES DE PESSEGUEIROS [PRUNUS PERSICA (L.)], EM PRESIDENTE PRUDENTE, ESTADO DE SÃO PAULO ${ }^{1}$
}

\author{
SÔNIA MARIA NALESSO MARANGONI MONTES ${ }^{2}$, ADALTON RAGA ${ }^{3}$, \\ APARECIDA CONCEIÇÃO BOLIANI ${ }^{4}$, JEFERSON LUIZ CARVALHO MINEIRO 5 , \\ PEDRO CÉSAR DOS SANTOS ${ }^{4}$
}

RESUMO-Objetivou-se, nesta pesquisa, estudar a ocorrência natural de ácaros fitófagos e predadores em diferentes cultivares de pessegueiro, no município de Presidente Prudente-SP, Brasil. O estudo foi realizado no período de dezembro de 2002 a fevereiro de 2006. Amostras quinzenais de 72 folhas foram coletadas ao acaso, de pessegueiros das cultivares Talismã, Doçura 2, Dourado 2, Tropical, Aurora 1 e Aurora 2. Coletou-se um total de 2.594 ácaros, sendo 2.092 fitófagos, 403 predadores e 99 de hábitos alimentares pouco conhecidos, com 35 espécies de ácaros de 16 famílias. Aculus fockeui ocorreu de maneira esporádica, não causando danos visíveis às plantas. A família Phytoseiidae apresentou a maior abundância e o maior número de indivíduos. O predador Euseius citrifolius foi o mais abundante. Não houve preferência dos ácaros nas cultivares de pessegueiro avaliadas.

Termos para indexação: Prunus persica, ácaros, ocorrência, similaridade, análise faunística.

\section{MITE DIVERSITY IN PEACH CULTIVARS [PRUNUS PERSICA (L.)], IN PRESIDENTE PRUDENTE, STATE OF SÃO PAULO, BRAZIL}

\begin{abstract}
This study aimed to investigate the occurrence of phytophagous and predator mites in peach cultivars in Presidente Prudente municipality, State of São Paulo, Brazil. The survey was conducted from December 2002 to February 2006. Leaf samples were collected fortnightly of 72 sheets of cultivars Talismã, Doçura 2, Dourado 2, Tropical, Aurora 1 and Aurora 2. It was recovered 2,594 mites, among 2,092 phytophagous, 403 predators and 99 of dietary habits few knowledge. It was collected 35 mite species belong to 16 families. Aculus fockeui occurred on a sporadic season, causing no visible damage to the plants. In the case of phytoseids, Euseius citrifolius was the most abundant predator species. No preference of mites was detected among the peach cultivars.
\end{abstract}

Index terms: Prunus persica, mites, occurrence, similary, faunistic analysis.

\section{INTRODUÇ̃̃O}

O lançamento de cultivares de pouca exigência em frio para superação da endodormência (BARBOSA et al., 1997) levou a expansão da área de cultivo de culturas de clima temperado, como o pessegueiro, para regiões com pouca disponibilidade de frio no inverno (BARBOSA et al., 2003), sendo observado seu deslocamento para outras regiões subtropicais e tropicais do interior paulista, como Marília, São Carlos, Brotas e Jaboticabal (PEREIRA et al., 2002).
No pessegueiro, os ácaros têm sido apontados como importantes pragas. Destroem os tecidos superficiais das folhas, o que causa perda do suco celular junto às primeiras camadas do tecido foliar, ocorrendo amarelecimento ou bronzeamento nas infestações mais severas, podendo haver redução qualitativa e quantitativa dos frutos (SALLES, 1998).

No Brasil, as espécies de ácaros que atacam pessegueiros são: ácaro-rajado (Tetranychus urticae Koch, 1836) (Tetranychidae), ácaro-vermelho (Panonychus ulmi Koch, 1836) (Tetranychidae) e ácaro-prateado (Aculus cornutus Banks, 1905)

${ }^{1}$ (Trabalho 101-09). Recebido em: 22-04-2009. Aceito para publicação em: 20-11-2009. Trabalho desenvolvido com auxílio à pesquisa da FAPESP (Processo n $05 / 55649-5$ ).

${ }^{2}$ Eng.Agr. PqC.Dra.APTA-Pólo Regional Alta Sorocabana Rodovia Raposo Tavares Km 561 Caixa 298 Presidente Prudente-SP,Brasil CEP 19015-970 e-mail soniamontes@apta.sp.gov.br

${ }^{3}$ Eng.Agr. PqC. Dr.Centro Experimental Central-Instituto Biológico Rodovia Heitor Penteado Km 3, Caixa 70 Campinas-SP, Brasil CEP-13001-970 e-mail adalton@biologico.sp.gov.br

${ }^{4}$ Eng.Agr. Prof.Dr.Faculdade de Engenharia de Ilha Solteira-UNESP Av. Brasil, 56 Ilha Solteira-SP,CEP 15.385-000 e-mail: boliani@agr.feis.unesp.br; santospc@agr.feis.unesp.br

${ }^{5}$ Biól. Dr. Centro Experimental Central-Instituto Biológico Rodovia Heitor Penteado Km 3 Caixa 70 Campinas-SP, Brasil. CEP13001-970 e-mail jmineiro@biologico.sp.gov.br 
(Eriophyidae) (FLECHTMANN, 1976; SANTACECÍLIA;SOUZA, 1997).

Em estudo sobre a população de ácaros predadores em pêssegos, Moraes et al. (1986) mencionaram Euseius concordis (Chant, 1959), Iphiseiodes zuluagai Denmark e Muma, 1972, Phytoseiulus macropilis (Banks, 1904) e Ricoseius loxocheles (DE LEON, 1965).

Os ácaros predadores são considerados efetivos no controle biológico de ácaros fitófagos, e sua ocorrência pode ser influenciada pelo manejo cultural das macieiras, afetando até o seu estabelecimento na planta quando liberada no campo (MONTEIRO, 2002).

Em vista da falta de registro de espécies de ácaros em pessegueiros, na região oeste do Estado de São Paulo, o presente trabalho objetivou estudar a composição da comunidade acarina, fundamental para compreender as interações entre as diversas espécies presentes nas diferentes cultivares a fim de subsidiar o manejo de pragas.

\section{MATERIAL E MÉTODOS}

O experimento foi realizado em pomar de pessegueiro instalado no Polo Regional da Alta Sorocabana, Agência Paulista de Tecnologia dos Agronegócios, da Secretaria da Agricultura e Abastecimento do Estado de São Paulo, Brasil, localizado no município de Presidente Prudente (UTM $7545288,76 \mathrm{~m} \mathrm{~N}, 459930,31 \mathrm{~m}$ E e altitude 424,29 $\mathrm{m})$. O estudo foi conduzido no período de dezembro/2002 a fevereiro/2006, em pomares de cinco anos de idade, instalados no espaçamento de $6,0 \times 3,0 \mathrm{~m}$. Foram analisadas as cultivares Dourado 2, Doçura 2, Tropical, Talismã, Aurora 2 e Aurora 1, oriundas de Programa de Melhoramento Genético do Instituto Agronômico de Campinas-IAC, com exigência de menos de 100 horas de frio. O porta-enxerto utilizado foi o Okinawa. Foi adotado o delineamento estatístico inteiramente casualizado, com uma planta por parcela. Os tratos culturais, durante a condução do estudo, foram os convencionais indicados para a cultura (PEREIRA et al., 2002), como: superação da dormência artificial realizada com Cianamida hidrogenada $(0,5 \%)+$ óleo mineral $(1 \%)$, raleio de frutos, adubações, controle de plantas espontâneas (Glyphosato a 0,5\% de i.a.), podas verde e seca, e irrigação por microaspersão.

As amostragens foram realizadas quinzenalmente, coletando-se 12 folhas dos terços superior, médio e inferior em cada face interna e externa da planta, totalizando 72 folhas por parcela. As amostras foram colocadas em sacos de papel e acondiciona- das em caixas de poliestireno contendo gelo para diminuir a atividade dos ácaros. As extrações foram realizadas no Laboratório de Sanidade e Qualidade Vegetal do Polo Regional Alta Sorocabana-APTA. As amostras eram colocadas individualmente em recipiente plástico e imersas durante 5 minutos em uma solução de álcool 70\% e agitadas para desalojamento dos ácaros. Desprezadas as folhas, passou-se a solução por uma peneira de $0,038 \mathrm{~mm}$, que foi recolhida em frascos de vidro com capacidade de $30 \mathrm{ml}$ para posterior triagem e identificação. Não foram amostradas folhas entre os meses de maio e agosto, devido à desfolha natural das plantas. Os ácaros coletados foram montados em meio de Hoyer. Uma amostra representativa das espécies encontradas foi depositada na Coleção de Referência de Ácaros Geraldo Calcagnolo, do Laboratório de Entomologia Econômica do Instituto Biológico (LEE/IB), em Campinas-SP.

A análise faunística dos dados coletados foi realizada utilizando-se dos índices de constância (acidental, acessória e constante) e dominância (acidental, acessória e dominante) para a determinação do status dos ácaros (raro, intermediário e constante), baseando-se no método proposto por Palma (1975). Para a análise da composição de espécies de ácaros nas diferentes cultivares de pessegueiros, foi utilizado o índice de similaridade de Morisita-Horn $\left(\mathrm{C}_{\mathrm{MH}}\right)$ (MAGURRAN, 1988), para estabelecer o grau de semelhança entre as diferentes cultivares nas folhas amostradas.

\section{RESULTADOS E DISCUSSÃO}

Registrou-se, no período, a ocorrência de 35 espécies de ácaros de 16 famílias (Quadros 1, 2 e 3). Foi coletado um total 2.594 ácaros, sendo 2.092 fitófagos, 403 predadores e 99 de hábitos alimentares pouco conhecidos.

Foi evidenciada, por meio de coleta em folhas de cultivares de pessegueiros, a ocorrência do ácaro fitófago Aculus fockeui (NALEPA, TROUESSART, 1891) (Eriophyidae) em todas as cvs. avaliadas, porém, em altas populações, apenas no mês de dezembro/2002 e, depois, em raras ocasiões (fevereiro e dezembro/2003 e novembro e dezembro/2005). Não foram observados sintomas do ataque de $A$. fockeui. Na cv. Dourado 2, foi encontrado em maior quantidade, representando $30,72 \%$ do total (797 indivíduos), e a menor, na cv. Talismã, com 3,24\% do total (84 indivíduos) (Tabela 1). Outro eriofídeo, Catarhynus sp., foi encontrado em número muito reduzido. A ocorrência de $A$. fockeui é reportada em várias regiões do mundo como principal ácaro do 
pessegueiro (KONDO; HIRAMATSU, 1999; FERREIRA; CARMONA, 1997; KUNUGI et al., 1993), ocasionando queda no peso dos frutos, diminuição no teor de açúcares (KUNUGI et al., 1993; KONDO; HIRAMATSU, 1999), desfolha e diminuição do vigor das plantas, resultando em baixa qualidade dos frutos no ano subsequente (KONDO; HIRAMATSU, 1999). Castagnoli e Nannelli (1987) encontraram altas densidades de $A$. fockeui em pomar de pêssego na Toscana, Itália, entretanto não observaram danos aparentes devido, provavelmente, ao controle pelos ácaros predadores.

$\mathrm{Na}$ família Tetranychidae, foram identificadas 5 espécies de ácaros (Quadro 1). O tetraniquídeo Tetranychus urticae foi encontrado em maior quantidade que os demais: 122 indivíduos no total das cultivares, tendo sido registrada maior incidência na cv. Talismã, com 2,20\% (57 indivíduos). O tenuipalpídeo Brevipalpus phoenicis (Geijskes) foi coletado em número reduzido nas cultivares Aurora 1, Aurora $2 \mathrm{e}$ Tropical $(0,12 \%, 0,39 \%$ e $0,12 \%$, respectivamente).

A ocorrência esporádica e o baixo nível populacional dos ácaros fitófagos, observados no presente estudo não chegaram a causar sinais visíveis de danos nas plantas, indicando não ser necessário o controle químico dos ácaros pelo baixo nível populacional ocorrido.

Em estudos realizados na Índia, Putatunda et al. (2002) observaram 90 espécies de ácaros em várias frutas, inclusive em pêssegos, com dominância de Tenuipalpidae e Tetranychidae, e de predadores Phytoseiidae e Stigmaeidae, resultados semelhantes aos encontrados no presente estudo, exceto para estigmeídeos, cuja ocorrência não foi registrada. $\mathrm{Na}$ região da Toscana, Itália, foram relatadas 20 espécies de ácaros (CASTAGNOLI; NANNELLI, 1987).

Dentre os predadores, constatou-se a presença das famílias Ascidae, Bdellidae, Cheyletidae, Erythraeidae e Phytoseiidae (Quadro 2). Registrou-se a maior riqueza de espécies e de números de indivíduos na família Phytoseiidae, sendo o ácaro E. citrifolius o mais abundante, com ocorrência em 5 das cultivares avaliadas (Tabela 1).

A ocorrência de predadores durante toda a avaliação deste estudo, principalmente de Euseius citrifolius Denmark e Muma, 1970, provavelmente, explique o baixo nível populacional de ácaros fitófagos. Na cv. Dourado 2, onde se registrou a maior percentagem de ocorrência de $A$. fockeui (30,72\%), observou-se também a menor percentagem de ocorrência do predador $E$. citrifolius $(1,35 \%)$, exercendo o controle biológico (Tabela 1). Resultado diferente foi observado por Ashihara et al. (2004), que encontraram injúrias nas folhas de pêssegos, redução no teor de açúcar dos frutos e queda de folhas pós-colheita, causadas pelo ácaro A. fockeui.

Em experimento conduzido no período de 1990/1991 em citros, em Presidente Prudente-SP, área hoje ocupada pelo pomar de pêssegos, objeto do presente estudo, Sato et al. (1994) identificaram seis espécies de fitoseídeos, com destaque para $I$. zuluagai, E. citrifolius e E. concordis, representando, respectivamente, $47,3 \%, 26,5 \%$ e $25,7 \%$ dos ácaros coletados. No presente estudo, E. citrifolius, E. concordis e I. zuluagai ocorreram com frequência de $13,49 \%, 1,73 \%$ e $0,08 \%$ do total de ácaros coletados.

Castagnoli e Nannelli (1987), na região da Toscana, relataram em pêssego a ocorrência de predadores das famílias Tydeidae, Orthotydeus kochi (OUDEMANS, 1929) e Pronematus ubiquitus (MCGREGOR, 1923), Phytoseiidae, Amblyseius andersoni (Chant, 1957) e Euseius stipulatus (ATHIAS-HENRIOT, 1960) e Stigmaeidae, Agistemus collyerae Gonzales-Rodriguez, 1963.

Com relação à diversidade populacional de ácaros fitófagos nas cultivares, a maior diversidade de espécies foi observada na cv. Tropical (6 espécies), seguida por Talismã, Aurora 1 e Aurora 2 (5 espécies), Dourado 2 e Doçura 2 (4 espécies). Os ácaros predadores não apresentaram a mesma tendência dos fitófagos, sendo observada na cv. Aurora 1 a maior riqueza, com 5 espécies (Tabela 2).

$\mathrm{Na}$ análise faunística das cvs. Talismã, Doçura 2 e Dourado 2, a maioria dos ácaros coletados recebeu o status de espécie rara, com 70\%,75\% e 66,67\%, respectivamente (Tabela 3). Nas cvs. Tropical, Aurora 1 e Aurora 2, a maioria dos ácaros coletados também recebeu o status de espécie rara, com $54,55 \%, 72,72 \%$ e $66,67 \%$, respectivamente (Tabela 4). T. urticae foi considerado intermediário para as cvs. Tropical, Talismã e Aurora 2, e raro para Doçura 2, Dourado 2 e Aurora 1. E. citrifolius foi considerado constante na maioria das cultivares, exceto na cv. Dourado 2, o que explica o elevado número do ácaro fitófago $A$. fockeui.

A. fockeui, considerado de ocorrência intermediária nas cultivares avaliadas, representou mais de $70 \%$ do total de ácaros capturados, tendo ocorrido de maneira abundante no início do levantamento (dezembro/2002), com as plantas em fase de formação, e de maneira esporádica ao longo do estudo. T. urticae, coletado no presente experimento em Aurora 2, e Talismã, juntamente com A. fockeui, são importantes ácaros fitófagos do pessegueiro. Resultados semelhantes foram obtidos por Ferreira e Carmona (1997) em pessegueiros de Portugal, onde observaram $41,6 \%$ de $A$. fockeui e $20 \%$ de 
Tetranychus cinnabarinus (Boisduval).

$\mathrm{Na}$ análise da composição de espécies de ácaros, nas diferentes cultivares de pessegueiros, observou-se que os índices foram muito próximos entre si. As cultivares com a composição da acarofauna mais similares entre si foram Aurora $2 \mathrm{com}$ Aurora 1 e Talismã, e Talismã com Doçura 2, com índice de 0,99 (Tabela 5).

Camporese e Duso (1996) relataram que ácaros-praga têm preferência por certas espécies de plantas em relação às outras, e geralmente fazem dis- tinção entre cultivares. Muitos dos aspectos químicos e da estrutura física da planta hospedeira podem afetar a taxa de crescimento de ácaros-praga (CAMPORESE; DUSO, 1996; GERSON et al., 2003). Entretanto, a similaridade da composição de espécies de ácaros nas diferentes cultivares de pessegueiros avaliadas mostra uma não preferência por determinada cultivar. Estes resultados servem de subsídio para o manejo do pessegueiro, considerando-se a grande importância da adoção de medidas de preservação de inimigos naturais, como os ácaros predadores.

QUADRO 1 - Ácaros fitófagos identificados em cultivares de pessegueiro. Presidente Prudente-SP.

\begin{tabular}{c|c|c}
\hline Ordem & Família & Gênero/Espécie \\
\hline Prostigmata & Diptilomiopidae Keifer, 1944 & Catarhynus sp. \\
\cline { 2 - 3 } & Eriophyidae Nalepa, 1898 & Aculus fockeui (Nalepa \& Trouessart, 1891) \\
\cline { 2 - 3 } & Tarsonemidae Kramer, 1877 & Fungitarsonemus sp. \\
& Tenuipalpidae Berlese, 1913 & Tarsonemus sp. \\
\cline { 2 - 3 } & & Brevipalpus phoenicis (Geijskes, 1939) \\
\cline { 2 - 3 } & Tetranychidae Donnadieu, 1875 & Tetranychus urticae Koch, 1836 \\
& & Eutetranychus sp. \\
& & Mononychellus planki (McGregor, 1950) \\
& & Oligonychus mcgregori (Baker \& Pritchard, 1953) \\
& & Oligonychus sp. \\
\hline
\end{tabular}

QUADRO 2 - Ácaros predadores identificados em cultivares de pessegueiro. Presidente Prudente-SP. $2002 / 2006$.

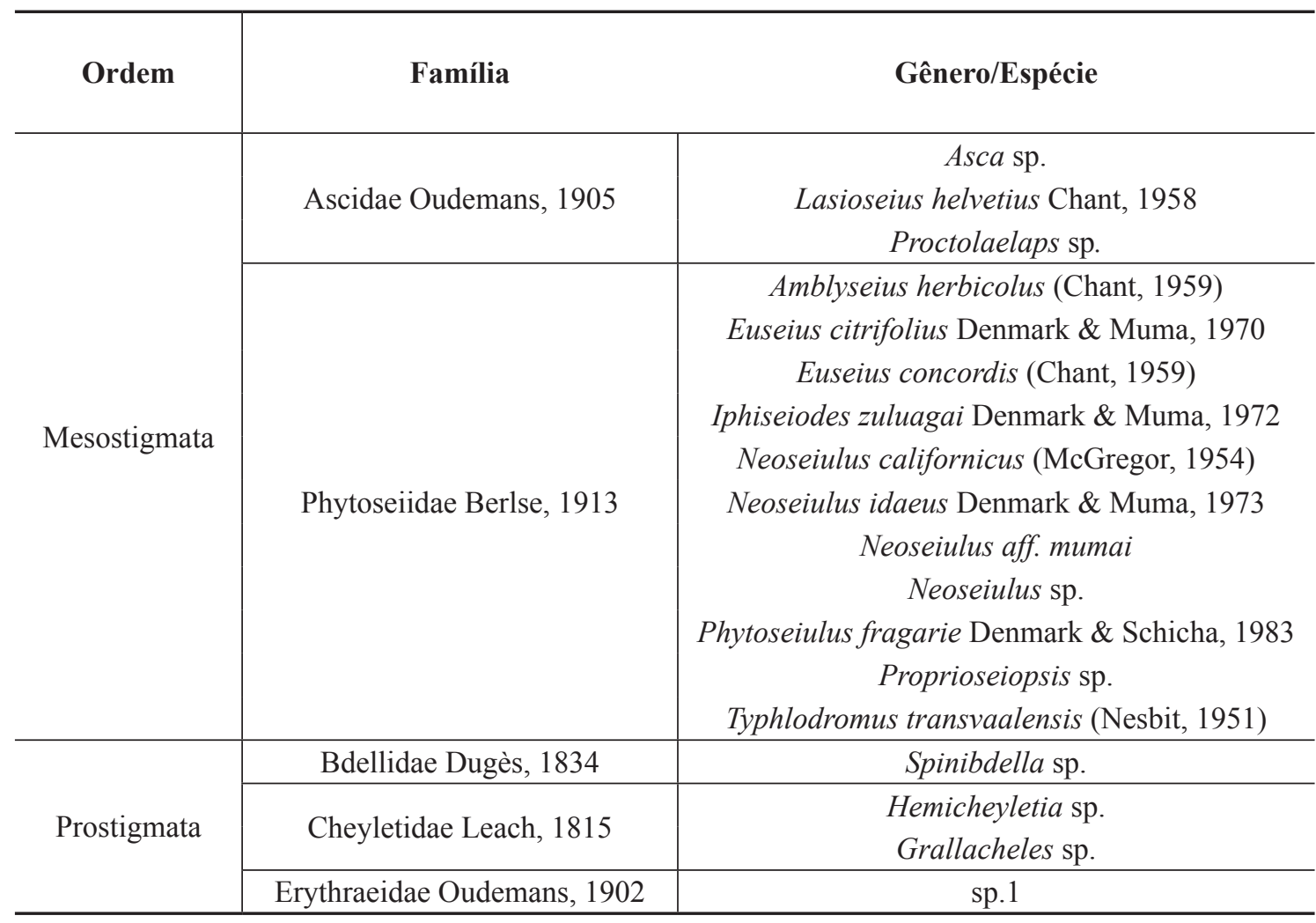


QUADRO 3- Ácaros de hábitos alimentares pouco conhecidos identificados em cultivares de pessegueiro. Presidente Prudente-SP. 2002/2006.

\begin{tabular}{c|c|c}
\hline Ordem & Família & Gênero/Espécie \\
\hline \multirow{2}{*}{ Astigmata } & Acaridae Latreille, 1802 & Tyrophagus sp. \\
& Winterschmidtiidae Oudemans, 1923 & Czenspinskia $\mathrm{sp}$. \\
\hline \multirow{2}{*}{ Cryptostigmata } & Cosmochthoniidae Grandjean, 1947 & Cosmochthonius $\mathrm{sp}$. \\
& Haplochthoniidae van der Hammen, 1959 & Haplochthonius $\mathrm{sp}$. \\
\hline \multirow{2}{*}{ Prostigmata } & Iolinidae & Homeopronematus $\mathrm{sp}$. \\
& Tydeidae Kramer, 1877 & Lorryia sp. \\
& Parapronematus acaciae Baker, 1965 \\
\hline
\end{tabular}

TABELA 1- Percentagem de ácaros fitófagos e fitoseídeos de maior ocorrência em relação ao total de ácaros coletados, em cultivares de pessegueiro. Presidente Prudente-SP. Dez/2002 a Fev/2006.

\begin{tabular}{lccccccc}
\hline \multirow{2}{*}{ Espécies } & Total ácaros & \multicolumn{5}{c}{ \% de ácaros em relação ao total coletado } \\
\cline { 5 - 8 } & coletados $\left(\mathrm{n}^{\mathrm{0}}\right)$ & Talismã & Doçura 2 & Dourado 2 & Tropical & Aurora 2 & Aurora 1 \\
\hline Fitófagas & & & & & & & \\
$\quad$ Aculus fockeui & 1909 & 3,24 & 12,45 & 30,72 & 7,59 & 14,19 & 8,87 \\
Mononychellus planki & 44 & 0,19 & 0,19 & 0,31 & 0,23 & 0,5 & 0,12 \\
Tetranychus urticae & 122 & 2,2 & 0,5 & 0,15 & 0,46 & 0,81 & 0,58 \\
Brevipalpus phoenicis & 16 &. &. &. & 0,12 & 0,39 & 0,12 \\
\hline Predadoras & & & & & & & \\
Amblyseius herbicolus & 1 &. &. &. &. &. & 0,04 \\
Euseius citrifolius & 350 & 1,58 &. & 1,35 & 3,51 & 2,81 & 2,85 \\
Euseius concordis & 45 & 0,31 & 1,39 & 0,35 & 0,15 & 0,42 & 0,35 \\
Neoseiulus sp. & 1 &. &. & 0,04 & 0,04 & 0,04 & . \\
\hline
\end{tabular}

TABELA 2- Distribuição percentual de espécies de ácaros ocorridas em cultivares de pessegueiro. Presidente Prudente-SP. Dez/2002 a Fev/2006.

\begin{tabular}{|c|c|c|c|c|c|c|}
\hline \multirow{2}{*}{ Espécies } & \multicolumn{6}{|c|}{ \% ocorrência } \\
\hline & Talismã & Doçura 2 & Dourado 2 & Tropical & Aurora 2 & Aurora 1 \\
\hline \multicolumn{7}{|l|}{ Fitófagas } \\
\hline Aculus fockeui & 39,25 & 84,11 & 90,47 & 39,63 & 72,16 & 66,47 \\
\hline Mononychellus planki & 2,34 & 1,30 & 0,23 & 19,63 & 2,55 & 0,87 \\
\hline Tetranychus urticae & 26,64 & 3,39 & 0,45 & 42,96 & 4,12 & 4,34 \\
\hline Homeopronematus sp. & 1,87 & 0,52 & 0,34 & 0,37 & 0,20 & 0,29 \\
\hline Brevipalpus phoenicis & & . & . & 1,11 & 1,96 & 0,87 \\
\hline Oligonychus sp. & & . & . & 0,37 & & \\
\hline Tarsonemus sp. & 0,47 &  & & 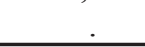 & & 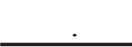 \\
\hline \multicolumn{7}{|l|}{ Predadoras } \\
\hline Amblyseius herbicolus & & & & & & 0,29 \\
\hline Euseius citrifolius & 19,16 & 9,38 & 3,97 & 2,96 & 14,31 & 21,39 \\
\hline Euseius concordis & 3,74 & 0,78 & 1,02 & 2,96 & 2,16 & 2,60 \\
\hline Iphiseiodes zuluagai & 0,93 & . & & . &  & \\
\hline Neoseiulus californicus & , & . & 0,11 & . & & . \\
\hline Neoseiulus aff.mumai & . & . & . & & 0,20 & . \\
\hline Neoseiulus idaeus & & $\cdot$ & . & 0,10 & . & . \\
\hline Neoseiulus sp. & & 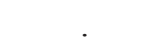 & . & 0,37 & . & 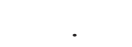 \\
\hline Typhlodromus transvaalensis & 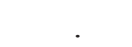 & 0,26 & . & . & . & 0,87 \\
\hline Hemicheyletia sp. & & & & & & 0,29 \\
\hline \multicolumn{7}{|l|}{ Hábito alimentar pouco conhecido } \\
\hline Lorryia sp. & & . & & 2,22 & 1,18 & \\
\hline Tyrophagus sp. & 5,14 & . & 2,84 & 4,07 & 0,78 & 2,02 \\
\hline Czenspinskia sp. & 0,47 & . & 0,57 & . & . & 0,29 \\
\hline Cosmochthonius sp. & & 0,26 & & & & \\
\hline
\end{tabular}


TABELA 3 - Análise faunística das espécies de ácaros em folhas de pessegueiro das cvs. Talismã, Doçura 2 e Dourado 2. Presidente Prudente-SP. 2002/2004.

\begin{tabular}{|c|c|c|c|c|c|c|}
\hline \multirow{2}{*}{ Espécies } & \multicolumn{2}{|c|}{ Talismã } & \multicolumn{2}{|c|}{ Doçura 2} & \multicolumn{2}{|c|}{ Dourado 2} \\
\hline & $\mathbf{N}$ & Status & $\mathbf{N}$ & Status & $\mathbf{N}$ & Status \\
\hline \multicolumn{7}{|l|}{ Eriophyidae } \\
\hline Aculus fockeui & 84 & $\mathbf{I}$ & 323 & $\mathbf{I}$ & 797 & I \\
\hline \multicolumn{7}{|l|}{ Tetranychidae } \\
\hline Mononychellus planki & 5 & $\mathbf{R}$ & 5 & $\mathbf{R}$ & 2 & $\mathbf{R}$ \\
\hline Tetranychus urticae & 57 & I & 13 & $\mathbf{R}$ & 4 & $\mathbf{R}$ \\
\hline \multicolumn{7}{|l|}{ Iolinidae } \\
\hline Homeopronematus sp. & 4 & $\mathbf{R}$ & 2 & $\mathbf{R}$ & 3 & $\mathbf{R}$ \\
\hline \multicolumn{7}{|l|}{ Phytoseiidae } \\
\hline Euseius citrifolius & 41 & $\mathrm{C}$ & 36 & $\mathrm{C}$ & 35 & I \\
\hline Euseius concordis & 8 & $\mathbf{R}$ & 3 & $\mathbf{R}$ & 9 & I \\
\hline Neoseiulus californicus & . & . & . & . & 1 & $\mathbf{R}$ \\
\hline Iphiseiodes zuluagai & 2 & $\mathbf{R}$ & . & . & . & . \\
\hline Typhlodromus transvaalensis & . & . & 1 & $\mathbf{R}$ & . & . \\
\hline \multicolumn{7}{|l|}{ Winterschmidtiidae } \\
\hline Czenspinskia sp. & 1 & $\mathbf{R}$ & . & . & 5 & $\mathbf{R}$ \\
\hline \multicolumn{7}{|l|}{ Acaridae } \\
\hline Tyrophagus sp. & 11 & $\mathbf{R}$ & . & . & 25 & $\mathbf{R}$ \\
\hline \multicolumn{7}{|l|}{ Tarsonemidae } \\
\hline Tarsonemus sp. & 1 & $\mathbf{R}$ & . & . & . & - \\
\hline \multicolumn{7}{|l|}{ Cosmochthoniidae } \\
\hline Cosmochthonius sp. & . & . & 1 & $\mathbf{R}$ & . & . \\
\hline
\end{tabular}

$\mathrm{N}$ : total de indivíduos

Status da espécie (C): comum ( I ) - intermediária (R): rara 
TABELA 4 - Análise faunística das espécies de ácaros em folhas de pessegueiro das cvs. Tropical, Aurora 2 e Aurora 1. Presidente Prudente-SP. 2002/2006.

\begin{tabular}{|c|c|c|c|c|c|c|}
\hline \multirow{2}{*}{ Espécies } & \multicolumn{2}{|c|}{ Tropical } & \multicolumn{2}{|c|}{ Aurora 2} & \multicolumn{2}{|c|}{ Aurora 1} \\
\hline & $\mathbf{N}$ & Status & $\mathbf{N}$ & Status & $\mathbf{N}$ & Status \\
\hline \multicolumn{7}{|l|}{ Eriophyidae } \\
\hline Aculus fockeui & 107 & $\mathbf{I}$ & 368 & $\mathbf{I}$ & 230 & $\mathbf{I}$ \\
\hline \multicolumn{7}{|l|}{ Tetranychidae } \\
\hline Mononychellus planki & 16 & $\mathbf{R}$ & 13 & $\mathbf{R}$ & 3 & $\mathbf{R}$ \\
\hline Oligonychus sp. & 1 & $\mathbf{R}$ & - & - & . & . \\
\hline Tetranychus urticae & 12 & I & 21 & I & 15 & $\mathbf{R}$ \\
\hline \multicolumn{7}{|l|}{ Tenuipalpidae } \\
\hline Brevipalpus phoenicis & 3 & $\mathbf{R}$ & 10 & $\mathbf{R}$ & 3 & $\mathbf{R}$ \\
\hline \multicolumn{7}{|l|}{ Iolinidae } \\
\hline Homeopronematus sp. & 1 & $\mathbf{R}$ & 1 & $\mathbf{R}$ & 1 & $\mathbf{R}$ \\
\hline \multicolumn{7}{|l|}{ Tydeidae } \\
\hline Lorryia sp. & 12 & $\mathbf{I}$ & 6 & $\mathbf{R}$ & . & . \\
\hline \multicolumn{7}{|l|}{ Phytoseiidae } \\
\hline Euseius citrifolius & 91 & C & 73 & $\mathbf{C}$ & 74 & $\mathbf{C}$ \\
\hline Euseius concordis & 5 & $\mathbf{R}$ & 11 & $\mathbf{R}$ & 9 & $\mathbf{R}$ \\
\hline Neoseiulus sp. & 1 & $\mathbf{R}$ & . & . & . & . \\
\hline Neoseiulus aff. mumai & - & . & 1 & $\mathbf{R}$ & - & - \\
\hline Typhlodromus transvaalensis & . & . & . & . & 1 & $\mathbf{R}$ \\
\hline Amblyseius herbicolus & - & • & - & - & 1 & $\mathbf{R}$ \\
\hline \multicolumn{7}{|l|}{ Acaridae } \\
\hline Tyrophagus sp. & 11 & $\mathbf{I}$ & 4 & $\mathbf{R}$ & 7 & $\mathbf{R}$ \\
\hline \multicolumn{7}{|l|}{ Winterschmidtiidae } \\
\hline Czenspinskia sp. & . & . & 2 & $\mathbf{R}$ & 1 & $\mathbf{R}$ \\
\hline \multicolumn{7}{|l|}{ Cheyletidae } \\
\hline Hemicheyletia sp. & . & . & . & . & 1 & $\mathbf{R}$ \\
\hline
\end{tabular}

$\mathrm{N}$ : total de indivíduos

Status da espécie (C): comum ( I ) - intermediária (R): rara 
TABELA 5 - Índice de Similaridade (Morisita-Horn) para composição de espécies de ácaros em folhas de pessegueiro, em Presidente Prudente-SP. 2002/2006.

\begin{tabular}{lcccccccc}
\hline & \multicolumn{7}{c}{ folhas de pessegueiro } \\
\cline { 2 - 9 } cultivares & número & número & \multicolumn{7}{c}{ Índice de Similaridade } \\
& espécies & indivíduos & Tropical & Aurora 2 & Talismã & Dourado 2 & Doçura 2 & $\begin{array}{c}\text { Aurora } \\
\text { Tropical }\end{array}$ \\
\cline { 2 - 9 } & 11 & 257 &. & 0,79 & 0,72 & 0,85 & 0,67 & 0,79 \\
Aurora 2 & 11 & 510 &. &. & 0,99 & 0,83 & 0,97 & 0,99 \\
Talismã & 10 & 214 &. &. &. & 0,75 & 0,99 & 0,96 \\
Dourado 2 & 9 & 881 &. &. &. &. & 0,69 & 0,89 \\
Doçura 2 & 8 & 384 & &. &. &. &. & 0,93 \\
Aurora 1 & 12 & 346 &. &. &. &. &. &. \\
\hline
\end{tabular}

\section{CONCLUSÕES}

1-Em cultura de pessegueiro no município de Presidente Prudente-SP, ocorrem 35 espécies de ácaros de 16 famílias.

2-Aculus fockeui ocorre de maneira esporádica, não causando danos visíveis às plantas.

3-A família Phytoseiidae apresenta a maior diversidade e abundância de ácaros predadores, com predominância de E. citrifolius.

4-As espécies de ácaros não mostram preferência por cultivares de pessegueiro.

\section{AGRADECIMENTOS}

À Dra. Denise Návia (EMBRAPA/CENARGEN), pela identificação do eriofí́deo, Aculus fockeui.

\section{REFERÊNCIAS}

ASHIHARA, W.; KONDO, A.; SHIBAO, M.; TANAKA, H.; HIEHATA, K.; IZUMI, K. ecology and control of eriophyid mites injurious to fruit trees in Japan. Japan Agricultural Research Quarterly, Tokyo, v.38, n.1, p. 31-41, 2004.

BARBOSA, W.; OJIMA, M.; CAMPO DALL'ORTO, F.A.; RIGITANO, O.; MARTINS, F.P.; SANTOS, F.P.; CASTRO, J.L. Melhoramento do pessegueiro para regiões de clima subtropical-temperado: realizações do Instituto Agronômico no período de 1950-1990. Campinas: Instituto agronômico, 1997. 22p. (Documentos IAC, 52).

BARBOSA, W.; POMMER, C.V.; RIBEIRO, M.D.;
VEIGA, R.F.A.; COSTA, A.A. Distribuição geográfica e diversidade varietal de frutíferas e nozes de clima temperado no estado de São Paulo. Revista Brasileira de Fruticultura, Jaboticabal, v.25, n.2, p.254-262, 2003.

CAMPORESE, P.; DUSO, C. Different colonization patterns of phytophagous and predatory mites (Acari: Tetranychidae, Phytoseiidae) on three grape varieties: a case study. Experimental and Applied of Acarology, Amsterdam, v.20, n.1, p.1-22, 1996.

CASTAGNOLI, M.; NANNELLI, R. Further observations on populations trend of mites in an experimental peach meadow orchard in central Italy. Redia, Firenze, v.70, n.2, p.121-133, 1987.

FERREIRA, M.A.; CARMONA, M.M. Acarofauna do pessegueiro em Portugal. Boletin de Sanidad Vegetal, Plagas, v.23, n.3, p.473-478, 1997.

FLECHTMANN, C.H.W. Ácaros de importância agrícola. São Paulo: Nobel, 1976. 150p.

GERSON, U.; SMILEY, R.L.; UCHOA, R. Mites (Acari) for pest control. Oxford: Blackwell Science, 2003. 539p.

KONDO, A.; HIRAMATSU, T. Analysis of peach tree damage caused by each silver mite, Aculus fockeui (Nalepa \& Trouessart). Japanese Journal of Applied Entomology and Zoology, Toshima-ku, v.43, n.4, p.189-193, 1999.

KUNUGI, Y.; TERAI, Y.; KATO, S. Seasonal occur- 
rence of peach silver mite, Aculus fockeui (Nalepa $\&$ Trouessart), on peach levels and their effects on fruit development. Proceedings of the Kanto Tosan Plant Protection Society, New York, v.40, n.2, p.269-271, 1993

MAGURRAN, A.E. Ecological diversity and its measurement. Princeton: University Press, 1988. 178p.

MORAES, G.J.; McMURTHY, J.A.; DENMARK, H.A. A catalog of the mite family Phytoseiidae: references to taxonomy, synonymy, distribution and habitat. Brasília: EMBRAPA-DDT, 1986. 353p.

MONTEIRO, L.B. Manejo integrado de pragas em macieiras no Rio Grande do Sul: uso de Neoseiulus californicus para o controle de Panonychus ulmi. Revista Brasileira de Fruticultura, Jaboticabal, v.24, n.2, p.395-405, 2002.

PALMA, S. Contribución al estudio de los sinoforos encontrados frente a la costa de Valparaiso. Aspectos ecologicos. In: SIMPOSIO LATINOAMERICANO OCEANOGRAFIA BIOLÓGICA, 2., 1975, Venezuela. Resumos... Venezuela: Universidade d'Oriente, 1975. p.119-133.
PEREIRA, F.M.; NACHTIGAL, J.C.; ROBERTO, S.R. Tecnologia para a cultura do pessegueiro em regiões tropicais e subtropicais. Jaboticabal: FUNEP, 2002. 61p.

PUTATUNDA, B.N.; MATHUR, R.B.; MATHUR, $\mathrm{S}$. Mites associates with some fruit trees in Hisar, Haryana. Journal of Agricultural Research, Lahore, v.36, n.2, p.88-95, 2002.

SALLES, L.A.B. Principais pragas e seu controle. In: MEDEIROS, C.A.B; RASEIRA, M.C. (Ed.). A cultura do pessegueiro. Brasília: SPI, 1998. p.205-242.

SANTA-CECÍLIA, L.V.C.; SOUZA, J.C. Pessegueiro e ameixeira: reconhecimento e manejo das principais pragas do pessegueiro. Informe Agropecuário Belo Horizonte, v.18, n.189, p.56-62, 1997.

SATO, M.E.; RAGA, A.; CERÁVOLO, L.C.; ROSSI, A.C.; POTENZA, M.R. Ácaros predadores em pomar cítrico de Presidente Prudente, Estado de São Paulo. Anais da Sociedade Entomológica do Brasil, Londrina, v.23, n.3, p.435-441, 1994. 